\title{
Magnetic field effect on quantum corrections to the low-temperature conductivity in metallic perovskite oxides
}

\author{
G. Herranz, F. Sánchez, and J. Fontcuberta \\ Institut de Ciència de Materials de Barcelona (ICMAB-CSIC), Campus UAB, Bellaterra 08193, Catalunya, Spain
}

\author{
V. Laukhin \\ Institut Català de Recerca i Estudis Avançats (ICREA), Barcelona, Spain and ICMAB-CSIC Campus UAB, Bellaterra 08193, \\ Catalunya, Spain \\ J. Galibert \\ Laboratoire National des Champs Magnétiques Pulsés, 143 avenue de Rangueil, 31432 Toulouse, Cedex 4, France \\ M. V. García-Cuenca, C. Ferrater, and M. Varela \\ Departament de Física Aplicada i Òptica, Universitat de Barcelona, Diagonal 647, Barcelona 08028, Catalunya, Spain
}

(Received 25 February 2005; revised manuscript received 2 May 2005; published 22 July 2005)

\begin{abstract}
The transport and magnetotransport properties of the metallic and ferromagnetic $\mathrm{SrRuO}_{3}(\mathrm{SRO})$ and the metallic and paramagnetic $\mathrm{LaNiO}_{3}$ (LNO) epitaxial thin films have been investigated in fields up to $55 \mathrm{~T}$ at temperatures down to $1.8 \mathrm{~K}$. At low temperatures both samples display a well-defined resistivity minimum. We argue that this behavior is due to the increasing relevance of quantum corrections to the conductivity (QCC) as temperature is lowered; this effect being particularly relevant in these oxides due to their short mean free path. However, it is not straightforward to discriminate between contributions of weak localization and renormalization of electron-electron interactions to the QCC through temperature dependence alone. We have taken advantage of the distinct effect of a magnetic field on both mechanisms to demonstrate that in ferromagnetic SRO the weak-localization contribution is suppressed by the large internal field leaving only renormalized electron-electron interactions, whereas in the nonmagnetic LNO thin films the weak-localization term is relevant.
\end{abstract}

DOI: $10.1103 /$ PhysRevB.72.014457

PACS number(s): 72.15.Rn, 72.80.Ng, 73.50.Fq

\section{INTRODUCTION}

In pure normal metals, the mean free path $l$ of the carriers is much larger than the Fermi wavelength $\lambda_{F}$. In this case, the semiclassical Boltzmann approach can successfully describe the transport properties of these metals. In this scenario, at $T<0.1 \theta_{D}$, where $\theta_{D}$ is the Debye temperature, the scattering of carriers by lattice imperfections leads to a lowtemperature resistivity which follows a $\rho(T)=\rho_{0}+A T^{n}$ dependence, where $\rho_{0}$ is the residual resistivity, $A$ is positive, and $n$ is a positive integer $(n=3-5$ for electron-phonon scattering, $n=2$ when electron-electron scattering dominates). However, as structural or composition disorder is increased, $n$ can change as well as the mean free path shrinking and eventually may become comparable to $\lambda_{F}$. In this situation, a fully quantum-mechanical treatment, accounting for the wavelike nature of the carriers, must be applied. This approach consists in adding some correcting terms to the lowtemperature conductivity, the so-called quantum corrections to the conductivity (QCC). ${ }^{1,2}$

QCC may arise from two different sources. First, there are effects coming from the self-interference of the wave packets as they are backscattered coherently by the impurities or other defects. This self-interference gives way to an additional scattering mechanism and to an enhancement of the resistivity. This picture is based on noninteracting carriers in a random potential. On the other hand, there is another con- tribution arising from the renormalization of the effective electron-electron interactions and the subsequent modification of the density of states at the Fermi energy. We refer to the former as the weak-localization (WL) and the latter as the renormalized electron-electron interaction (REEI) quantum corrections. Both contributions lead to an enhancement of resistivity as the temperature is decreased and can explain the presence of minima in the resistivity-temperature curves in disordered metals. However, it is not straightforward to ascertain whether WL or REEI is the most relevant contribution to QCC only through the analysis of the temperature dependence of the resistivity. Difficulty arises because for a two-dimensional (2D) system both effects lead to a $\Delta \sigma$ $\sim \ln T$ contribution to the conductivity whereas in the 3D limit, $\Delta \sigma \sim T^{1 / 2}$ or $\Delta \sigma \sim T^{p / 2}$ with $p=2-3$ for REEI or WL contributions, respectively. ${ }^{1,2}$ The narrow temperature range where these effects become observable and the identical (in the $2 \mathrm{D}$ case) or similar (in the 3D case) temperature behaviors do not allow a simple distinction between the two different physical sources of QCC. In general, in the 3D regime, the REEI contribution to QCC dominates over WL whereas in $2 \mathrm{D}$ both contributions are of comparable magnitude. However, as reviewed by Lee and Ramakrishnan, ${ }^{1}$ the observation of REEI and WL contributions to the temperature dependence of the conductivity in $3 \mathrm{D}$ systems have been reported. Instead, the field dependence of the lowtemperature resistivity is radically different, ${ }^{1}$ and in prin- 
ciple, it can unambiguously determine the nature of QCC. Indeed, by applying a magnetic field the WL term is suppressed, since the field destroys the wave coherence and, thus, the self-interference effects are reduced and the resistance is decreased. It then follows a negative magnetoresistance. Instead, the effect of the field on the REEI contribution leads to a positive magnetoresistance originated by the spin splitting of electrons in a magnetic field and by orbital effects. ${ }^{1}$

QCC have been proposed to explain the low-temperature transport properties of nonmagnetic disordered systems such as doped semiconductors and metallic alloys. ${ }^{1}$ Negative magnetoresistance (MR) has been observed in these systems, following the $\sqrt{H}$ law predicted by the theory in the 3D limit, consistent with a suppression of WL by the applied field. However, the situation could be different in some magnetic systems, in which the presence of a large internal magnetic field might suppress WL and then leave alone the REEI contribution. For instance, recently Manyala $e t$ al. have reported a positive MR at low temperature for the $\mathrm{Fe}_{1-y} \mathrm{Co}_{\mathrm{y}} \mathrm{Si}$ system, and they attributed it to the effect of the applied field on the REEI contribution. ${ }^{3}$ Several analyses of the low-temperature transport properties through QCC have also been performed in ferromagnetic oxide compounds. In particular, there are recent reports on the $\mathrm{La}_{2 / 3} \mathrm{Sr}_{1 / 3} \mathrm{MnO}_{3}$ system ${ }^{4,5}$ suggesting the presence of these quantum effects. However, these experiments are rather intricate, due to the difficulty in separating the contribution coming from the effects of the field on the QCC from other MR sources. The high magnetoresistance of manganites and their strong sensitivity to magnetic disorder are a hindrance to a further verification of QCC by performing extended experiments on magnetoresistance. We can partially overcome these shortcomings using $\mathrm{SrRuO}_{3}$ (SRO) samples, since in this itinerant ferromagnetic perovskite, the weaker sensitivity to magnetic disorder results in a weaker MR.

In this paper we show magnetotransport data of a SRO film (metallic ferromagnet) and a $\mathrm{LaNiO}_{3}$ (LNO) film (metallic paramagnet). Both films showed a resistivity upturn at low temperature which can be explained by the presence of QCC. The relevance of these quantum effects is justified by the closeness of the mean free path $l$ to the Fermi wavelength $\lambda_{F}$. Knowing that the Fermi energy of SRO is $E_{F} \approx 2 \mathrm{eV}$, and the band effective mass is $m^{*} \approx 3.7 m_{e},{ }^{6}$ we can estimate $\lambda_{F}$ $\approx 4.5 \AA$. The mean free path $l$ of the analyzed SRO film $(t=3.5 \mathrm{~nm})$ is estimated from $\sigma=n e^{2} \tau / m^{*}=n e^{2} l \lambda_{F} / h$, with $n \approx 2 \times 10^{22} \mathrm{~cm}^{-3}{ }^{7}$ For our SRO sample, $\sigma$ $\approx 2 \times 10^{3} \Omega^{-1} \mathrm{~cm}^{-1}$ at $5 \mathrm{~K}$, so $l \approx 5-6 \AA$ at low temperature, and we have $k_{F} l=\left(2 \pi / \lambda_{F}\right) l=O(1)$. In LNO, the carrier density is $n \approx 1.7 \times 10^{22} \mathrm{~cm}^{-3}$. Since in the analyzed LNO film $(t=240 \mathrm{~nm}) \quad \sigma \approx 3 \times 10^{3} \Omega^{-1} \mathrm{~cm}^{-1}$ at $5 \mathrm{~K}$, we have that $l \approx 6 \AA$ at low temperature. On the other hand, for LNO it is known that $E_{F} \approx 0.21 \mathrm{eV}$, and $m^{*} \approx 11 m_{e},{ }^{9}$ and we obtain $\lambda_{F} \approx 8 \AA$. Thus, again, $k_{F} l=O(1)$.

Indeed, in previous reports, we have established on solid grounds a relationship between QCC and structural disorder in SRO thin films. ${ }^{10,11}$ Through the analysis of the present MR data, we conclusively show that the WL is suppressed in the SRO film by the internal strong magnetic field, whereas it is not the case in the LNO film. This result emphasizes the role of the magnetic field in the quantum corrections. The presence of a large internal field in the ferromagnetic SRO sample is responsible for the suppression of the WL contribution, and only the REEI contribution is relevant in that case.

\section{EXPERIMENT}

It is well known that SRO has an unusual high magnetocrystalline anisotropy ${ }^{12}(>2 \mathrm{~T})$ and a strong anisotropic magnetoresistance. ${ }^{13}$ By applying very strong fields, up to $55 \mathrm{~T}$, we can minimize contributions to MR coming from other sources different from that of the effect of the external field on QCC. We have performed magnetotransport measurements in pulsed high magnetic fields [Laboratoire National des Champs Magnétiques Pulsés (CNRS, Toulouse)] of an epitaxial SRO film grown by pulsed laser deposition on a nominally exact (100) $\mathrm{SrTiO}_{3}$ substrate. Details on preparation conditions and structural properties of our SRO films can be found elsewhere. ${ }^{14-16}$ The measured samples had an almost rectangular shape with typical dimensions of about $\sim 3.5 \times 2.5 \mathrm{~mm}^{2}$. Here we will show, as a typical example, data collected for a film $t=3.5 \mathrm{~nm}$ thick. Magnetoresistance experiments were carried out using the standard ac fourprobe method in a pulsed magnetic field (applied perpendicular to the film) of up to $55 \mathrm{~T}$ (pulse decay time $0.18 \mathrm{~s}$ ) in the temperature range from 1.8 to $280 \mathrm{~K}$. (In this paper we will concentrate on and discuss only the low-temperature part related to the localization phenomena.) Four in-line electrical contacts to the films were made using Pt wires of $20 \mu \mathrm{m}$ in diameter glued with graphite paste. An alternating current $(10 \mu \mathrm{A}, 23 \mathrm{kHz})$ was injected. We have also verified that in the $0.1-50 \mu \mathrm{A}$ range, at low temperature ( $T$ $\leqslant 4.2 \mathrm{~K}$ ), the response is Ohmic. A lock-in amplifier with a time constant of $100-300 \mu$ s was used to detect the signal across the potential leads.

The magnetotransport data of the LNO film were obtained in a Physical Property Measurement System (PPMS, Quantum Design), with fields of up to $9 \mathrm{~T}$ applied also perpendicular to the film. Transport measurements were performed with contacts made on previously deposited Au. The epitaxial LNO film $(t \approx 240 \mathrm{~nm})$ was grown on a $\mathrm{LaAlO}_{3}$ substrate by the pulsed laser deposition technique. ${ }^{17}$

\section{RESULTS AND DISCUSSION}

Figure 1 shows the temperature dependence of the resistivity measured at $H=0 \mathrm{~T}$ of SRO (a) and LNO (b) films; the resistivity upturn at low temperature is well visible in both cases. These data have been fitted by the least-square minimization method to QCC (including both WL and REEI contributions) in both the 3D and 2D limits. In the first case (3D), the corresponding $T$ dependence is given by ${ }^{1}$

$$
\rho(T)=\frac{1}{\sigma_{0}+a_{1} T^{p / 2}+a_{2} T^{1 / 2}}+b T^{2}
$$

where the $a_{1} T^{p / 2}$ term accounts for the 3D WL contribution and the $a_{2} T^{1 / 2}$ term stands for the 3D REEI correction; the 

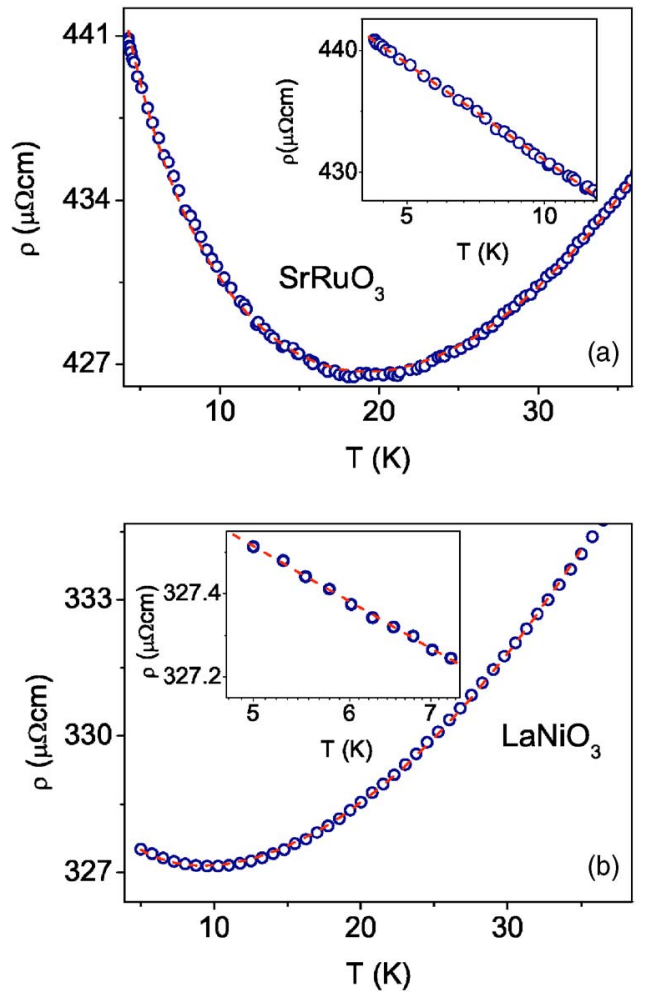

FIG. 1. (Color online) Temperature dependence of the resistivity of the SRO (a) and LNO (b) films. Dashed line is the result of the fit using Eq. (2). Insets: zoom of the low-temperature region of resistivity plotted in a semilogarithmic scale.

$a_{1} T^{p / 2}$ term depends on the length scale imposed by the inelastic collisions on the quantum coherence length of the carriers. If electron-electron interactions are dominant, $p=2$, whereas $p=3$ if electron-phonon scattering prevails. ${ }^{1} \mathrm{We}$ have also added the Boltzmann term $\left(b T^{2}\right)$ accounting for the classical low-temperature dependence of the resistivity and assumed the Mattiessen rule to hold. For the 2D case, both QCC contributions give similar temperature dependence: ${ }^{1}$

$$
\rho(T)=\frac{1}{\sigma_{0}+a \ln T}+b T^{2} .
$$

We have fitted the data of Fig. 1, using all these models. It turned out that the 3D and the 2D models give similar reliability factors of fitted parameters and thus a distinction is unfair. For the sake of simplicity, only the fitting to expression (2) is shown in Fig. 1 (dashed line through the data). In the corresponding insets, we display a zoom of the lowtemperature region, to visualize the quality of the $\ln T$ behavior in this temperature range. As a means to evaluate the quality of the fittings, Fig. 2 shows the deviation plots, defined as $\Delta \rho_{n}=\left[\rho_{\text {fitting }}(T)-\rho(T)\right] / \rho(T)$, as a function of the temperature for fittings to expressions (1) and (2). The plotted fittings in the 3D limit correspond to an $a_{1} T^{3 / 2}$ inelastic length cutoff defined by electron-phonon collisions; qualitatively similar results are found when an $a_{1} T$ is considered (electron-electron collisions). We see that it is not easy to discriminate whether the $3 \mathrm{D}$ or $2 \mathrm{D}$ limit is the more appropriate only through the analysis of the $T$ dependence. More-
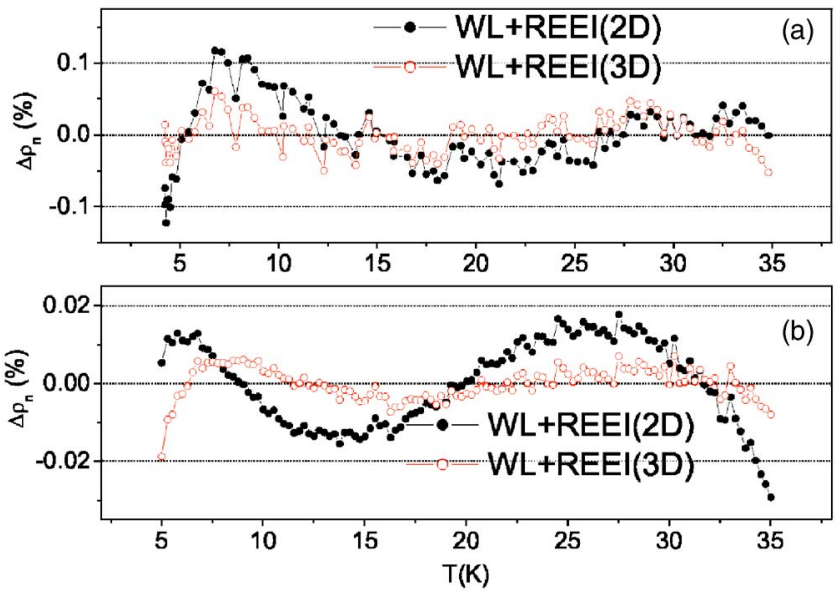

FIG. 2. (Color online) Temperature dependence of the deviation $\Delta \rho_{n}=\left[\rho_{\text {fitting }}(T)-\rho(T)\right] / \rho(T)$ corresponding to fittings to expressions (1) (3D limit) and (2) (2D limit) for the SRO (a) and the LNO (b) films.

over, it is important to find an alternative way to evaluate the contributions of the WL and REEI physical mechanisms to QCC. This is of special interest in the 2D limit, since both 2D REEI and WL contributions have the same $\ln T$ behavior. Hopefully, as pointed out above, the analysis of the highfield MR may be an alternative method to discriminate unequivocally whether the quantum effects come from REEI or WL effects.

In Fig. 3(a) (inset) we show the low-temperature dependence of the electrical resistance of the SRO film measured at fields of $H=0$ and $48 \mathrm{~T}$. We observe that the application of high field $(H=48 \mathrm{~T})$ lowers the resistivity, i.e., the total magnetoresistance is negative. The field dependence of the magnetoresistance $\mathrm{MR},[R(H)-R(0)] / R(0)$, for different temperatures is shown in Fig. 4. This large negative magnetoresistance is likely related to spin disorder effects. More significantly, a detailed inspection of the lowesttemperature region of the curves in the inset of Fig. 3(a) reveals that the upturn of the resistance measured under field is slightly shifted to a higher temperature with respect to the data recorded at zero field. This feature is more easily observed when we shift the curves by subtracting the resistance at the minimum from the raw resistance data [Fig. 3(a), main panel]. After this subtraction it becomes evident that the resistance minimum under field is noticeably shifted to higher temperatures and the resistance below $T_{\min }$, down to the lowest temperature, is visibly larger under the $48 \mathrm{~T}$ field when it is compared with data recorded at zero field. This fact is a clear indication that the QCC effects are enhanced when a magnetic field is applied. Therefore, we infer that there is a positive MR contribution, which is masked by the (larger in magnitude) negative MR (probably coming from the suppression of magnetic disorder by the applied field).

Possible origins of the positive MR contribution include orbital effects. The orbital magnetoresistance $\mathrm{MR}_{\text {orb }}$ can be estimated to be $\mathrm{MR}_{\text {orb }} \approx\left(H / n e \rho_{0}\right)^{2}$ where $n$ is the carrier density and $\rho_{0}$ the residual resistivity. Using appropriate $\left(n, \rho_{0}\right)$ values, it turns out that $\mathrm{MR}_{\text {orb }} \approx 0.001 \%$ for fields of a few tens of teslas and thus this contribution is negligible in 

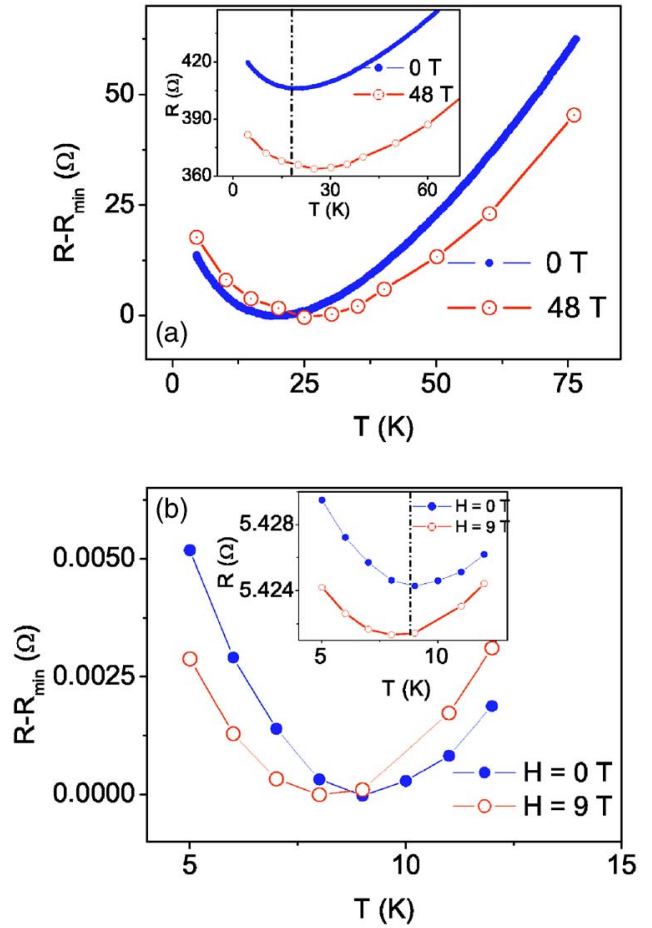

FIG. 3. (Color online) Insets: Resistance versus temperature data measured at zero field as well as at field of $48 \mathrm{~T}$ for typical SRO (a) and at $9 \mathrm{~T}$ for LNO (b) films. Main panels: temperature dependence of the resistance measured after subtraction of the resistance at minimum at zero field as well as at field of $48 \mathrm{~T}$ for SRO (a) and at $9 \mathrm{~T}$ for LNO (b) films.

SRO. Similar conclusions were derived in Refs. 18 and 19. Instead, we suggest that the effect of field on the QCC arising from the REEI gives such positive contribution to the MR. As discussed by several authors, spin-splitting effects in an interacting electron gas can lead to a positive magnetoresistance. ${ }^{1,2}$ In order to have this mechanism operative, the condition $g \mu_{B} H \gg k_{B} T$ must be satisfied. ${ }^{1}$ In our case, we have performed the experiments at a temperature of order $\sim 10 \mathrm{~K}$, i.e., $k_{B} T \sim 1 \mathrm{meV}$. Since the maximum applied field is of about $55 \mathrm{~T}$, we have $g \mu_{B} H \sim 6 \mathrm{meV}$. Therefore, at these high fields, a positive magnetoresistance due to spinsplitting effects might be detected.

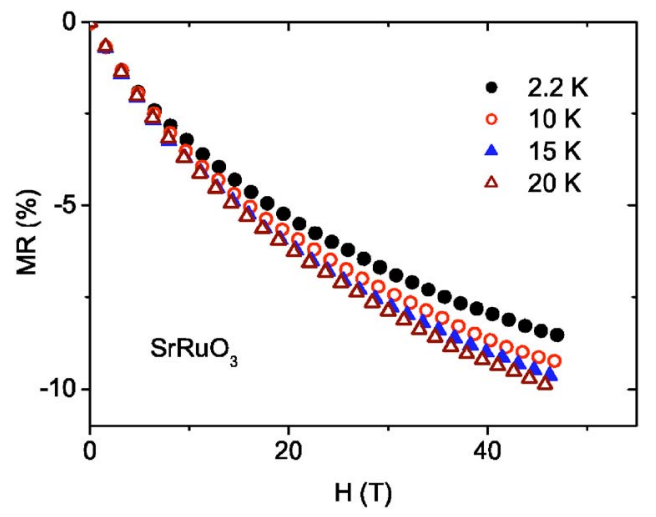

FIG. 4. (Color online) The field dependence of the magnetoresistance $\mathrm{MR}=[R(H)-R(0)] / R(0)$ at different temperatures is plotted for the same SRO film as in Fig. 3(a) $(t=3.5 \mathrm{~nm})$.
As mentioned above, we had previously (Refs. 10 and 11) analyzed the correlation between structural disorder and QCC in SRO films. From the analysis of the temperature dependence of zero-field data we argued that the REEI was likely to be the dominant contribution to QCC. This suggestion is consistent with the present high-field MR measurements showing that the relevant contribution to QCC should come from the REEI term. The fact that SRO is a ferromagnetic material might explain why the WL term is suppressed.

Now, it appears noteworthy to explore the magnetotransport properties of a disordered nonmagnetic perovskite oxide. In this case, the WL term should not be suppressed due to the lack of any internal magnetic field and its presence should be reflected in the magnetotransport properties. An optimal candidate to explore this issue is LNO, which is a metallic perovskite oxide with no long-order magnetic correlations. In the past, QCC have been put forward to explain the low-temperature magnetotransport properties of LNO., ${ }^{9,20}$ It has to be noted that the samples under that study were polycrystalline in nature, and that a significant contribution to resistivity coming from grain boundaries could not be avoided. In our case, we have performed transport measurements on a high-quality epitaxial LNO thin $(t=240 \mathrm{~nm})$ film grown on a $\mathrm{LaAlO}_{3}$ substrate ${ }^{17}$ and thus, we expect that any grain boundary contribution should be minimized.

Figure 3(b) (inset) shows the temperature dependence of the resistance of the LNO film measured at $H=0$ and $9 \mathrm{~T}$. The data indicate that the total MR is negative. However, in contrast to the SRO case, the minimum of resistance under field is shifted slightly toward lower temperatures. To compare better both curves we proceed as previously: we have shifted the curves by subtracting the resistance value at the minimum [see Fig. 3(b), main panel]. Data in this figure clearly reveal that the resistance upturn, measured under field, is shifted to a lower temperature and down to the lowest temperature, the resistance is reduced when compared to its zero-field value. That means that the application of the magnetic field has suppressed partially the effects of QCC. One can, therefore, assign tentatively this effect to a suppression of the wave coherence of the carriers by the field, i.e., a field suppression of WL. To clarify further this issue, the field dependence of the electrical resistivity of the LNO film, showing a minimum near $10 \mathrm{~K}$ [see Fig. 1(b)], was analyzed at different temperatures. As observed in Fig. 5(a) [see also inset in Fig. 3(b)], the magnetoresistance, defined as MR $=[R(H)-R(0)] / R(0)$, is negative, but the magnitude decreases monotonically with increasing temperature, and above $\sim 15 \mathrm{~K}$ it becomes negligible. Indeed, the MR measured at $20 \mathrm{~K}$ is practically washed out, i.e., the negative MR vanishes at temperatures somewhat above the resistance upturn. One can assign this remarkable temperature dependence to a negative MR arising from destruction of weak localization by the application of magnetic field. For large enough fields, the field dependence of the electrical conductivity at low temperatures should follow either a $\sqrt{H}$ dependence (3D limit) or a $\ln H$ dependence (2D limit), whereas for small fields an $H^{2}$ dependence has been predicted. ${ }^{1}$ We have verified that the experimental $\Delta \sigma=\sigma(H)-\sigma(0)$ data are better fitted when plotted against $H^{1 / 2}$ as shown in Fig. 6 and, therefore, we assume henceforth that we are in the 3D limit. We 

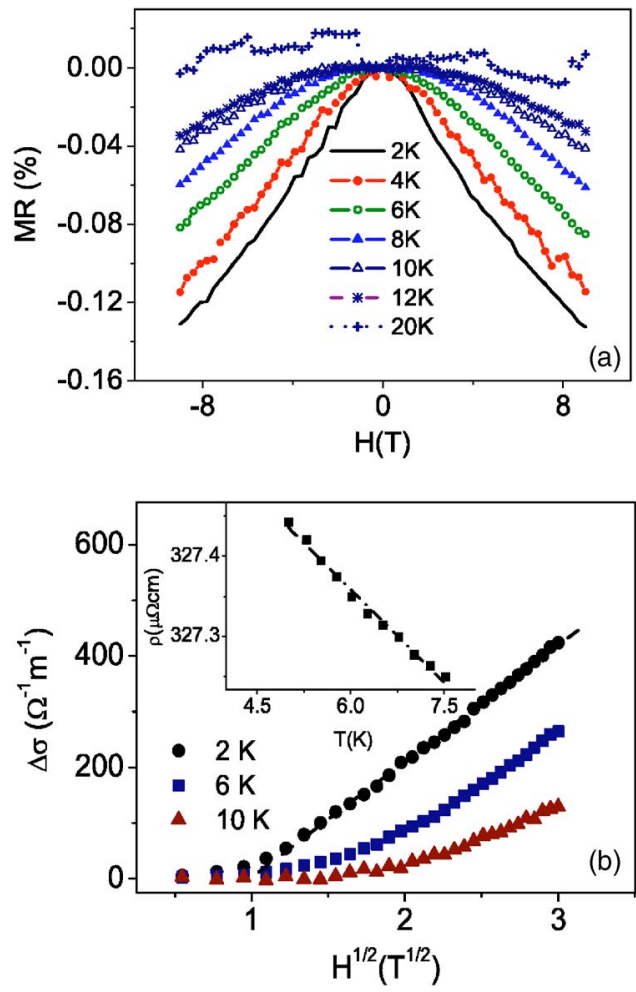

FIG. 5. (Color online) (a) The field dependence of the magnetoresistance $\mathrm{MR}=[R(H)-R(0)] / R(0)$ at different temperatures is plotted for a LNO film with thickness $t=240 \mathrm{~nm}$. (b) The magnetoconductance change $\Delta \sigma=\sigma(H)-\sigma(0)$ is plotted versus $H^{1 / 2}$ at different temperatures for the same LNO film. The dashed line through the $2 \mathrm{~K} \Delta \sigma(T)$ data is a fitting using Eq. (3). Inset: The $T$ dependence of $\rho$ is fitted to Eq. (5) (dash-dotted line) in the range $5 \leqslant T$ $\leqslant 7.5 \mathrm{~K}$.

see in Fig. 5(b) that $\Delta \sigma$ measured at $2 \mathrm{~K}$ follows an $H^{1 / 2}$ law above $\sim 1.5 \mathrm{~T}$. This figure also shows $\Delta \sigma$ vs $H^{1 / 2}$ measured at higher temperatures. We see that as the temperature increases, the magnetoresistance becomes smaller and the $R(H)$ curves deviate progressively from the $H^{1 / 2}$ dependence.

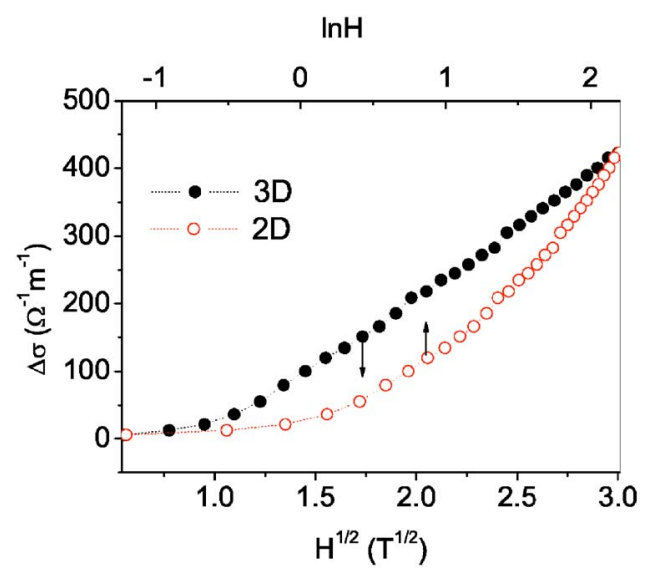

FIG. 6. (Color online) The magnetoconductance change $\Delta \sigma$ $=\sigma(H)-\sigma(0)$ is plotted versus $H^{1 / 2}$ (3D limit) and versus $\ln H(2 \mathrm{D}$ limit) in the same range of $H$ values for the same LNO film of Fig. 5.
This can be explained by the gradual washing out of the weak-localization effect as $T$ increases.

The field-induced conductivity change (negative magnetoresistance) $\Delta \sigma=\sigma(H)-\sigma(0)$ resulting from the suppression of weak localization by a magnetic field is given by ${ }^{1}$

$$
\begin{gathered}
\Delta \sigma=m \sqrt{H}, \\
m=\frac{e^{2}}{2 \pi^{2}} \sqrt{e / \hbar} f(x)
\end{gathered}
$$

where $x=\hbar /\left(4 e H l_{\text {in }}{ }^{2}\right) ; l_{\text {in }}$ is the inelastic mean free path, which is temperature dependent, and $f(x)$ is a smooth function of $x$ (see Ref. 1). We have observed that the $\Delta \sigma$ data were best fitted against an $H^{1 / 2}$ dependence (see the fitting in the range $1.5 \leqslant H \leqslant 9 \mathrm{~T}$ of the curve measured at $2 \mathrm{~K}$ [Fig. $5(\mathrm{~b})]$ ). This case corresponds to the asymptotic value of $x \ll 1$ and, therefore, $f(x)=0.605,{ }^{1}$ and thus the theoretical value of $m$ is $\approx 291 \Omega^{-1} \mathrm{~m}^{-1} \mathrm{~T}^{-1 / 2}$. This value compares nicely with the experimental one $m \approx 208.5 \Omega^{-1} \mathrm{~m}^{-1} \mathrm{~T}^{-1 / 2}$, extracted from the fit of data in Fig. 5(b).

On the other hand, as shown in Ref. 1, the condition $x \ll 1$ means that $l_{\text {in }} \gg \sqrt{\hbar / 4 e H} \approx 13 H^{-1 / 2} \mathrm{~nm}$, i.e., in the range of the analyzed fields $(H=1.5-9 \mathrm{~T}), l_{\text {in }} \gg 4-10 \mathrm{~nm}$. Indeed, phase-coherence lengths of around $15 \mathrm{~nm}$ have been determined in $\mathrm{LaNi}_{1-x} \mathrm{Co}_{x} \mathrm{O}_{3}(x=0.25-0.4) .{ }^{9}$ Here we recall that the thickness of the analyzed sample is $t \approx 240 \mathrm{~nm}$, which is far above the estimated inelastic cutoff length $l_{\text {in }}$ at $\sim 10 \mathrm{~K}$ and then the assumption of a 3D limit is consistent. Therefore, it follows from experimental data and these cross checks that the magnetoconductance can be well explained by the suppression of weak localization by the applied field.

The previous conclusions are further verified for the $T$ dependence of the resistivity. The temperature dependence of the WL contribution to QCC is given by ${ }^{1}$

$$
\Delta \sigma_{\mathrm{WL}}=\frac{e^{2}}{\pi^{3} l_{\mathrm{in}}},
$$

where $l_{\text {in }}$ is the $T$-dependent inelastic cutoff length. To get an estimate of $l_{\text {in }}$ we have fitted the $\rho(T)$ of the LNO sample by using

$$
\rho(T)=\frac{1}{\sigma_{0}+a_{\mathrm{WL}} T^{p / 2}}, \quad p=2,
$$

where $\Delta \sigma_{\mathrm{WL}}=a_{\mathrm{WL}} T$, in the range $5 \leqslant T \leqslant 7.5 \mathrm{~K}$; notice that only the WL contribution is considered [see the inset of Fig. 5(b)]. A value $a_{\mathrm{WL}} \sim 100 \Omega^{-1} \mathrm{~m}^{-1} \mathrm{~K}^{-1}$ has been obtained from this fitting. From Eqs. (4) and (5) it follows that

$$
l_{\text {in }}=\frac{e^{2}}{\pi^{3} a_{\mathrm{WL}} T} .
$$

Substituting values, we find that $10<l_{\text {in }}<80 \mathrm{~nm}$ in the range $1 \leqslant T \leqslant 10 \mathrm{~K}$. This result agrees with the above assertion according to which $l_{\text {in }} \gg 4-10 \mathrm{~nm}$. Recalling that the film thickness is $t \approx 240 \mathrm{~nm}$, this confirms the $3 \mathrm{D}$ regime of this sample (see also Fig. 6) and, thus, the analysis of $\rho(T)$ considering WL contribution is consistent with that of the $H$ dependence of the MR. 
Before concluding we would like to address the significance of the observed Ohmic behavior of measurements on $\mathrm{SrRuO}_{3}$ films. Indeed, current-voltage $I(V)$ characteristics have been measured at $4 \mathrm{~K}$ for all samples and it is found that up to the maximum applied voltage $(<40 \mathrm{mV})$ all samples display an Ohmic behavior. In the weak-localization regime, electron-electron correlations renormalize the density of states at the Fermi level and, as shown in Refs. 1 and 2 , the single-particle density of states is given by $g(\varepsilon)$ $=g\left(E-E_{F}\right)=g_{0}\left[1+|\varepsilon / \Delta|^{0.5}\right]$ where $\Delta$ is the so-called correlation gap. The presence of a finite $\Delta$ implies that the conductance of the sample may not be linear in the applied voltage. Indeed, early tunneling experiments performed on the closely related $\mathrm{LaNi}_{x} \mathrm{Co}_{1-x} \mathrm{O}_{3}$ oxide revealed a non-Ohmic behavior, with a correlation gap of about $\Delta \approx 5 \mathrm{meV}$ for $x$ $>0.75 .^{20}$ Our experimental finding of an Ohmic behavior at least up to $40 \mathrm{mV}$ in measurements performed at $4 \mathrm{~K}$ could be interpreted as an indication of a thermal-smearing effect in the conductance or that the correlation gap is substantially smaller that $40 \mathrm{mV}$. However, as signaled by Raychaudhuri et al. ${ }^{20}$ alternative explanations could not be disregarded. For instance, the coexistence of inelastic scattering process at the barriers below the electrical contacts would also give rise to the Ohmic behavior. Of course, this discussion is meaningful only if the measured voltage-dependent conductance reflects exclusively the density of states $g(\varepsilon)$ in the film. The available data do not allow extracting, at this moment, more conclusive information on the significance of the observed Ohmic behavior.

In summary, quantum corrections to the conductivity successfully describe the low-temperature electrical conductivity of the metallic perovskites SRO and LNO. The presence of QCC is fingerprinted by the occurrence of resistivity minima at some temperature $T_{\min }$. In general, these quantum effects could arise from two different sources: weak localization and renormalization of electron-electron interactions. Here, we have shown that the WL term is suppressed in the ferromagnetic SRO by the strong internal field, whereas in LNO this term is relevant. We attribute this difference to the fact that LNO is nonmagnetic; in absence of a large internal magnetic field, weak localization prevails. This is manifested by a negative magnetoresistance which disappears at temperatures above $T_{\text {min }}$. Instead, in SRO the WL term is canceled, the REEI contribution prevails, and a positive contribution to magnetoresistance follows.

Financial support by the CICYT of the Spanish Government (Projects No. MAT2002-04551-C03 and No. MAT2003-4161) and FEDER is acknowledged.
${ }^{1}$ P. A. Lee and T. V. Ramakrishnan, Rev. Mod. Phys. 57, 287 (1985).

${ }^{2}$ B. L. Altshuler and A. G. Aronov, in Electron-Electron Interaction in Disordered Conductors, edited by A. L. Efros and M. Pollak (North-Holland, Amsterdam, 1985).

${ }^{3}$ N. Manyala, Y. Sidis, J. F. DiTusa, G. Aeppli, D. P. Young, and Z. Fisk, Nature (London) 404, 581 (2000).

${ }^{4}$ M. Auslender, A. E. Kar'kin, E. Rozenberg, and G. Gorodetsky, J. Appl. Phys. 89, 6639 (2001).

${ }^{5}$ M. Ziese, Phys. Rev. B 68, 132411 (2003).

${ }^{6}$ P. B. Allen, H. Berger, O. Chauvet, L. Forro, T. Jarlborg, A. Junod, B. Revaz, and G. Santi, Phys. Rev. B 53, 4393 (1996).

${ }^{7}$ L. Klein, J. S. Dodge, C. H. Ahn, G. J. Snyder, T. H. Geballe, M. R. Beasley, and A. Kapitulnik, Phys. Rev. Lett. 77, 2774 (1996).

${ }^{8}$ N. Gayathri, A. K. Raychaudhuri, X. Q. Xu, J. L. Peng, and R. L. Greene, J. Phys.: Condens. Matter 10, 1323 (1998).

${ }^{9}$ K. P. Rajeev and A. K. Raychaudhuri, Phys. Rev. B 46, 1309 (1992).

${ }^{10}$ G. Herranz, B. Martínez, J. Fontcuberta, F. Sánchez, C. Ferrater, M. V. García-Cuenca, and M. Varela, Phys. Rev. B 67, 174423 (2003).

${ }^{11}$ G. Herranz, F. Sánchez, B. Martínez, J. Fontcuberta, M. V. García-Cuenca, C. Ferrater, M. Varela, and P. Levy, Eur. Phys. J. B 40, 439 (2004).
${ }^{12}$ L. Klein, J. S. Dodge, C. H. Ahn, J. W. Reiner, L. Mieville, T. H. Geballe, M. R. Beasley, and A. Kapitulnik, J. Phys.: Condens. Matter 8, 10111 (1996).

${ }^{13}$ D. B. Kacedon, R. A. Rao, and C. B. Eom, Appl. Phys. Lett. 71, 1724 (1997).

${ }^{14}$ G. Herranz, B. Martínez, J. Fontcuberta, F. Sánchez, M. V. García-Cuenca, C. Ferrater, and M. Varela, Appl. Phys. Lett. 82, 85 (2003).

${ }^{15}$ F. Sánchez, M. V. García-Cuenca, C. Ferrater, M. Varela, G. Herranz, B. Martínez, and J. Fontcuberta, Appl. Phys. Lett. 83, 902 (2003).

${ }^{16}$ F. Sánchez, G. Herranz, I. C. Infante, J. Fontcuberta, M. V. García-Cuenca, C. Ferrater, and M. Varela, Appl. Phys. Lett. 85, 1981 (2004).

${ }^{17}$ F. Sánchez, C. Ferrater, C. Guerrero, M. V. García-Cuenca, and M. Varela, Appl. Phys. A: Mater. Sci. Process. 71, 59 (2000).

${ }^{18}$ L. Klein, A. F. Marshall, J. W. Reiner, C. H. Ahn, T. H. Geballe, M. R. Beasley, and A. Kapitulnik, J. Magn. Magn. Mater. 188, 319 (1998).

${ }^{19}$ G. Herranz, F. Sánchez, M. V. García-Cuenca, C. Ferrater, M. Varela, B. Martínez, and J. Fontcuberta, J. Magn. Magn. Mater. 272-276, 517 (2004).

${ }^{20}$ A. K. Raychaudhuri, K. P. Rajeev, H. Srikanth, and N. Gayathri, Phys. Rev. B 51, 7421 (1995). 